\title{
Transmission Ratio Distortion Due to the $b l$ Gene in Table Beet
}

\author{
Diane Austin ${ }^{1}$ \\ Department of Statistics, 1210 W. Dayton Street, University of Wisconsin, Madison, WI 53706 \\ I.L. Goldman ${ }^{2}$ \\ Department of Horticulture, 1575 Linden Drive, University of Wisconsin, Madison, WI 53706
}

AdDitional INDEX wORDs. segregation distortion, meiotic drive, red beet, garden beet, betalain pigment, Beta vulgaris ssp. vulgaris.

\begin{abstract}
The $b l$ gene conditions a blotchy phenotype (irregular sectors of red and white root color) in table beet (Beta vulgaris ssp. vulgaris). Segregation of the $b l$ gene was found to be consistent with a single recessive gene, however, some evidence for a departure from a single gene model was observed when blbl plants were used as females. In this report, segregation of the $b l$ gene was examined in greater detail in $10 \mathrm{~F}_{2}$ populations derived from crosses of red blotchy-rooted females (genotype $b l b l$, denoted blotchy) with red-rooted males ( $B l B l$, denoted red,), and $10 \mathrm{~F}_{2}$ populations derived from the reciprocal cross. In $b l b l \times B l B l$ crosses, the proportion of red-rooted progeny was greater than 0.75 in seven of the crosses, and was significantly greater $(P=0.005)$ in three crosses. A test for heterogeneity was significant, indicating that the proportion of red-rooted progeny differed significantly in these 10 crosses. In $B l B l$ x $b l b l$ crosses, the proportion of red-rooted progeny was $<0.75$ in seven of the crosses and there were no significant departures from the expected 3:1 ratio in any of the individual crosses. However, a pooled estimate of the segregation ratio showed a significant $(P<0.01)$ departure from the 3:1 ratio (pooled estimate $=0.71$ ). These data demonstrate transmission ratio distortion at the $b l$ locus when $b l b l$ plants are used as both females and males in matings with wild type plants, but the degree of distortion is greater when $b l b l$ plants are used as females. Ratio distortion in such crosses may be due to a variety of factors, including increased transmission of the $b l$ gene through female or male gametes depending on the direction of the cross, reduced fitness of maternally derived $b l b l$ progeny, epigenetic phenomena, increased fitness of paternally derived $b l b l$ progeny, or linkage of the $b l$ gene to viability genes.
\end{abstract}

Meiotic drive is defined as any alteration in the meiotic process that results in the excess transmission of one allele over its alternative from a heterozygote (Ganetzky, 1999; Sandler and Novitski, 1957). Meiotic drive in plants has been associated with B chromosomes (Jones, 1991), chromosome knobs (Buckler et al., 1999), gametocidal genes (Tsujimoto and Tsunewaki, 1985), and paramutation (Hollick et al., 1997). A manifestation of meiotic drive is segregation distortion, which is also known as transmission ratio distortion or segregation ratio distortion. This phenomenon has been noted widely in plant genetic studies (see review by Zamir and Tadmor, 1986), but its mechanism in most cases remains poorly understood.

Lyttle (1991, 1993) defined two specific types of meiotic drive: genic and chromosomal. In genic meiotic drive, one member of a pair of heterozygous alleles is transmitted to progeny in excess of the expected Mendelian proportions of 50\%. In chromosomal meiotic drive, one member of a pair of heteromorphic chromosomes is likewise excessively transmitted. Both types occur in plants (Lyttle, 1993), although some examples of meiotic drive in plants involve interactions between drive loci and chromosome structure (Buckler et al., 1999). Segregation distorters may increase in frequency in a population regardless of their fitness, and do so at the expense of their allelic counterparts (Lyttle, 1991).

Zamir and Tadmor (1986) reported 54\% of 114 random loci from the genera Lens Mill., Capsicum L., and Lycopersicon Mill. exhibited segregation ratio distortion in populations derived from inter-

Received for publication 3 July 2000. Accepted for publication 16 Jan. 2001. We thank D. Nicholas Breitbach for assistance with crosses and field operations. The cost of publishing this paper was defrayed in part by the payment of page charges. Under postal regulations, this paper therefore must be hereby marked advertisement solely to indicate this fact.

${ }^{1}$ Predoctoral trainee.

${ }^{2}$ Associate professor and corresponding author; email: ilgoldma@ facstaff.wisc.edu. specific crosses. The proportion of distorted ratios was only $13 \%$ in populations derived from intraspecific crosses with these genera. Aberrant segregation in these crosses was attributed to linkage between marker genes and either pre or post zygotic viability genes. During development of molecular marker linkage maps, many workers have observed segregation distortion at molecular marker loci (Patterson et al. 1990). In most cases, these distorted loci occur more frequently in populations derived from interspecific crosses.

Watson and Goldman (1997) described a gene $b l$ conditioning a blotchy phenotype (irregular sectors of red and white root color) in table beet (Beta vulgaris ssp. vulgaris). Segregation of $b l$ was found to be consistent with a single recessive gene in four $\mathrm{BC}_{1}$ and two $\mathrm{F}_{2}$ populations; however, some evidence $(P<0.10$ and $P<0.08)$ for departures from a single gene model were observed when $b l b l$ plants were used as females. In these cases, an excess of red-rooted progeny was present in $b l b l \times B l B l$ matings. Further examination of the maternal and paternal transmission of $b l$ was warranted. In this report, segregation of the $b l$ gene was examined in $10 \mathrm{~F}_{2}$ populations derived from red blotchy-rooted females (genotype $b l b l$, denoted blotchy) and red-rooted males (genotype $B l B l$, denoted red, crosses 1-10), and $10 \mathrm{~F}_{2}$ populations derived from the reciprocal cross (crosses 11-20).

\section{Materials and Methods}

Seeds of the blotchy (genotype at primary color loci was $R R Y Y b l b l$ ) and red genotypes (genotype at primary color loci was $R R Y Y B l B l$ ) used in this investigation were planted in the table beet breeding nursery at the University of Wisconsin during May 1996. At harvest (August 1996), roots were washed, trimmed, and vernalized for 8 weeks. Vernalized roots were planted in the greenhouse in November and used in crosses according to standard protocols. All matings were made between fertile plants. $F_{1}$ 
Table 1. Goodness-of-fit of the observed $\mathrm{F}_{2}$ segregation data from 20 crosses. The expected ratio for all crosses is 3:1.

\begin{tabular}{|c|c|c|c|c|c|c|}
\hline \multirow[b]{2}{*}{ Cross } & \multicolumn{2}{|c|}{ Red } & \multicolumn{2}{|c|}{ Blotchy } & \multirow[b]{2}{*}{$\chi^{2}(\mathrm{df}=1)$} & \multirow[b]{2}{*}{$P$} \\
\hline & $\mathrm{No}^{\mathrm{z}}$ & Proportion & No. & Proportion & & \\
\hline & \multicolumn{6}{|c|}{ Blotchy $x$ red } \\
\hline 6 & 117 & 0.975 & 3 & 0.025 & 32.40 & $<0.001^{*}$ \\
\hline 8 & 144 & 0.911 & 14 & 0.089 & 21.95 & $<0.001^{*}$ \\
\hline 4 & 62 & 0.873 & 9 & 0.127 & 5.75 & 0.017 \\
\hline 7 & 98 & 0.867 & 15 & 0.133 & 8.29 & $0.004^{*}$ \\
\hline 9 & 83 & 0.783 & 23 & 0.217 & 0.62 & 0.433 \\
\hline 3 & 68 & 0.764 & 21 & 0.236 & 0.09 & 0.759 \\
\hline 2 & 64 & 0.762 & 20 & 0.238 & 0.06 & 0.802 \\
\hline 10 & 78 & 0.743 & 27 & 0.257 & 0.03 & 0.865 \\
\hline 5 & 63 & 0.724 & 24 & 0.276 & 0.31 & 0.578 \\
\hline \multirow[t]{2}{*}{1} & 52 & 0.642 & 29 & 0.358 & 5.04 & 0.025 \\
\hline & \multicolumn{6}{|c|}{ Red x blotchy } \\
\hline 13 & 80 & 0.784 & 22 & 0.216 & 0.64 & 0.423 \\
\hline 11 & 90 & 0.783 & 25 & 0.217 & 0.65 & 0.419 \\
\hline 15 & 51 & 0.750 & 17 & 0.250 & 0.00 & 1.000 \\
\hline 20 & 66 & 0.710 & 27 & 0.290 & 0.81 & 0.369 \\
\hline 17 & 70 & 0.707 & 29 & 0.293 & 0.97 & 0.324 \\
\hline 12 & 51 & 0.699 & 22 & 0.301 & 1.03 & 0.311 \\
\hline 16 & 57 & 0.687 & 26 & 0.313 & 1.77 & 0.183 \\
\hline 19 & 53 & 0.679 & 25 & 0.321 & 2.07 & 0.150 \\
\hline 14 & 72 & 0.661 & 37 & 0.339 & 4.65 & 0.031 \\
\hline 18 & 57 & 0.626 & 34 & 0.374 & 7.42 & 0.007 \\
\hline
\end{tabular}

${ }^{\mathrm{z} N u m b e r}$ of progeny in each cross.

* Significant at $P=0.005$.

seeds were harvested in April and planted in the breeding nursery in May 1997. No differences in viability of $F_{1}$ populations were noted. Due to the possibility of some self-pollination during the formation of $F_{1}$ populations, care was taken to identify obvious hybrids, based on heterosis for size and expression of nonblotchy root color, during the production of $F_{1}$ plants in $1997 . F_{1}$ roots were harvested in August, vernalized as described above, and planted in the greenhouse in November for crossing. Although most red beet germplasm is self-incompatible, the blotchy mutant was detected in selfcompatible breeding material in $N$ cytoplasm and was therefore selffertile. $F_{1}$ plants were self-pollinated during Winter 1997-98. $F_{2}$ populations were planted in May 1998 in the breeding nursery. Plants were harvested in August 1998 and scored for the red root or red blotchy root phenotypes. Chi-square goodness-of-fit tests were performed for each cross. The chi-square tests have the form $\chi^{2}{ }_{1}=$ $\left[\left(n_{\text {red }}-0.75 n\right)^{2} / 0.75 n\right]+\left[\left(n_{\text {blotchy }}-0.25 n\right)^{2} / 0.25 n\right]$, where $n$ is the number of progeny in each cross, and $\mathrm{n}_{\text {red }}$ and $\mathrm{n}_{\text {blotchy }}$ are the numbers of red-rooted and blotchy-rooted progeny in each cross. Because there were 10 comparisons for each type of cross, an adjusted $P$ level of 0.005 was used for individual crosses. The usual $P=0.05$ was used for all other statistical tests. The proportions of red and blotchy roots and chi-square statistics for each cross are listed in Table 1. A test of heterogeneity was performed to determine if crosses 1 to 10 could be pooled. $\chi_{\text {heterogeneity }}^{2}$ is defined as $\chi_{\text {total }}^{2}-\chi_{\text {pooled }}^{2}$, where $\chi_{\text {total }}^{2}$ is the sum of the $\chi^{2}$ values from the individual crosses, and $\chi_{\text {pooled }}^{2}=$ $\left[\left(\mathrm{T}_{\text {red }}-0.75 \mathrm{~T}\right)^{2} / 0.75 \mathrm{~T}\right]+\left[\left(\mathrm{T}_{\text {blotchy }}-0.25 \mathrm{~T}\right)^{2} / 0.25 \mathrm{~T}\right]$, where $T$ is the total number of progeny from 10 crosses, and $\mathrm{T}_{\text {red }}$ and $\mathrm{T}_{\text {blotchy }}$ are the corresponding totals of red an blotchy-rooted progeny.

\section{Results and Discussion}

When a trait segregates as a single recessive gene, it is expected that the trait will be expressed in 0.25 of the progeny in the $\mathrm{F}_{2}$ generation. In the crosses considered herein, 0.25 of the progeny were expected to have red blotchy roots and 0.75 were expected to have red roots. Figure 1 shows the proportion of redrooted beets from each cross, ordered from the largest proportion of red beets to the smallest.

A larger than expected proportion of red-rooted plants was observed in crosses 1 to 10 (blotchy $x$ red) and a smaller than expected proportion of red beets was observed in crosses 11 to 20 (red $x$ blotchy). In crosses 1 to 10 (blotchy $x$ red), seven of the crosses had greater than 0.75 red-rooted progeny, with a range from $0.642-0.975$ (Table 1 and Fig. 1). Significant departures from the expected segregation ratio were detected in three of these crosses. In all three cases (crosses 6, 8, and 7), there were more red beets (and fewer red blotchy) than expected. Cross 4 had a larger proportion of red beets than cross 7 ( $0.873 \mathrm{vs} .0 .867)$, but because there were fewer progeny in cross 4 , the chi-square statistic was not significant at the adjusted $P$ level $(0.005)$. By contrast, cross 1 had only 0.642 red-rooted progeny, which was the second smallest proportion of red-rooted progeny in all 20 crosses. For crosses 1 to $10, \chi^{2}$ heterogeneity $=74.54-24.68=49.86 ; P$ value $\left(\chi^{2}\right.$, $9 \mathrm{df})=<0.0001$. There was strong evidence that the segregation ratios in crosses 1 to 10 differed from each other; therefore, it was not appropriate to pool these 10 crosses.

In the reciprocal crosses (crosses 11 to 20 ; red x blotchy), seven of the 10 crosses had fewer than 0.75 red-rooted progeny, with a range from 0.626 to 0.784 . There were no significant departures (at $P=0.005)$ from the expected 3:1 ratio when each cross was considered individually. A test of heterogeneity was also performed for these 10 crosses: $\chi_{\text {heterogeneity }}^{2}=20.01-7.69=12.32 ; P$ value $\left(\chi^{2}\right.$, $9 \mathrm{df})=0.196$. Because the test for heterogeneity was not significant, the segregation ratios in these crosses were similar enough to be pooled. The pooled estimate was 0.71 , and it was significantly less than the expected ratio $\left(\chi_{\text {pooled }}^{2}=7.69, P=0.0056\right)$. 


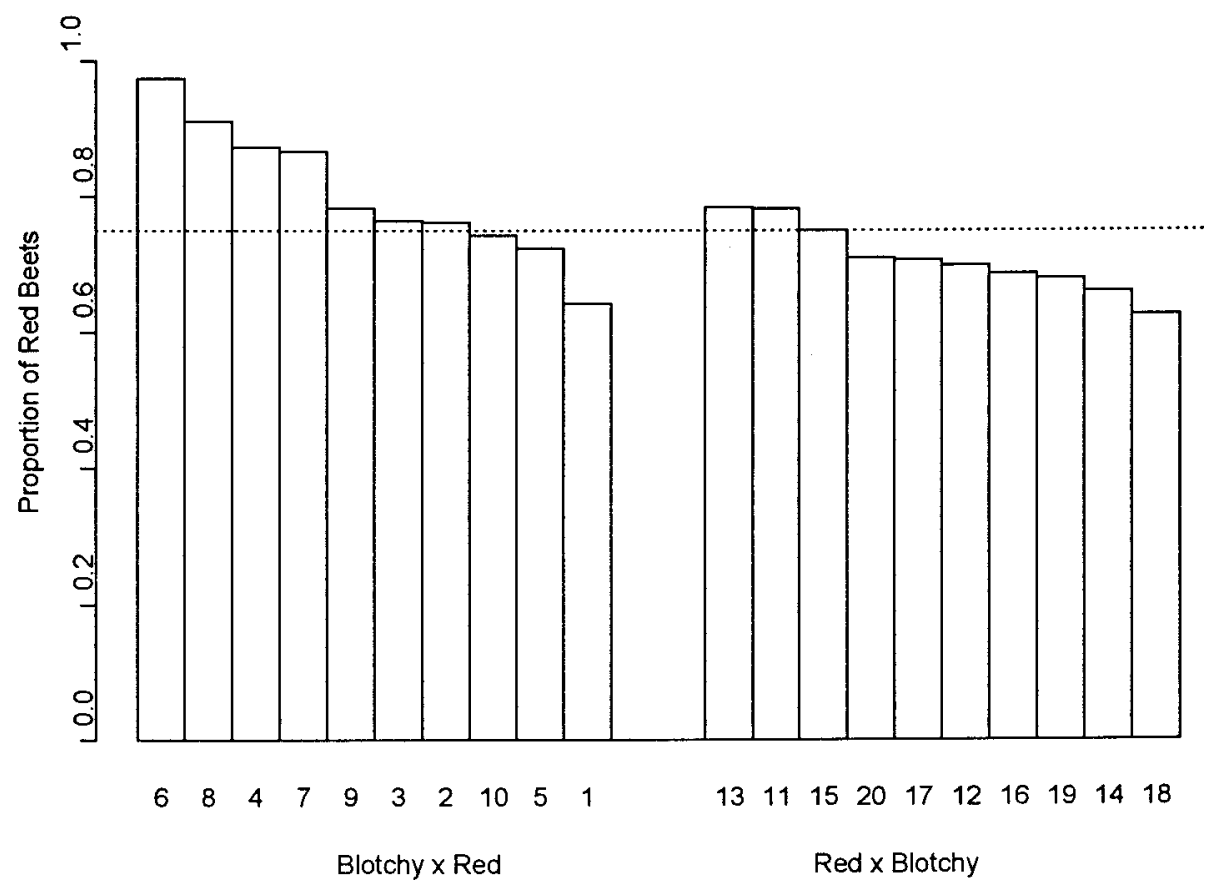

noted when $b l b l$ plants were used as both males and females in matings with wild type plants, however, the distortion was significantly greater when $b l b l$ plants were used as females.

In plants, recent studies of paramutation and chromosome knobs have provided some explanations for mechanisms affecting segregation distortion. Paramutation is an allelic interaction that results in heritable changes in gene expression. Paramutable heterozyous loci will exhibit heritable conversions of one allele into another, thereby potentially altering segregation ratios. Paramutable loci have been described in maize (Zea mays L.), tomato (Lycopersicon esculentum Mill.), petunia (Petunia $\times$ hybrida Hort. Vilm.-Andr.), and tobacco (Nicotiana tabacum L.) (reviewed in Hollick et al., 1997). Mechanisms for the nature of the heritable change and the allelic interactions have been advanced (Hollick et al., 1997). Chromosome knobs are heterochromatic blocks consisting of

Fig. 1. Proportion of red beets from 20 crosses. The cross number is given below each bar, and the expected ratio (0.75) is illustrated by the dotted line.

As a final test for the differences between the two groups of crosses, the proportions of red beets for crosses 1 to 10 were compared to the proportions for crosses 11 to 20 using a WilcoxonMann-Whitney test. The proportions in the two groups were significantly different $(P=0.026)$.

Two of the crosses described in Watson and Goldman (1997) were similar to crosses 1 to 10 in this study. In the $\mathrm{F}_{2}$ generation, both crosses had larger proportions of red roots than expected (0.766 and 0.770). The chi-square goodness-of-fit statistics for these crosses were 2.69 and 2.95 , respectively, with corresponding $P$ values of 0.10 and 0.08 (note that the second chi-square value and the $P$ values have been corrected from Table 1 in Watson and Goldman, 1997). While these results were not statistically significant, increased proportions of red-rooted progeny were also present in these two crosses.

The remaining four crosses discussed in Watson and Goldman (1997) used the reciprocal cross (red x blotchy) in the first generation, but used a backcross (to the blotchy parent) for the second generation. Chi-square goodness-of-fit tests of a 1:1 ratio were all nonsignificant $\left(\chi^{2}=0.14\right.$ to $1.09, P=0.30$ to 0.70$)$. While it is interesting that the chi-square values for the $\mathrm{BC}_{1}$ crosses were smaller than the values for the $\mathrm{F}_{2}$ crosses, it is not possible to determine whether they differed because of a difference in the parental cross or because of a difference in the genetic model.

Meiotic drive and the corresponding segregation distortion resulting from it are only beginning to be understood at the molecular level. Recently, the product of the segregation distorter (Sd) gene from Drosophila melanogaster was identified and correlated to defects in nuclear function and chromatin condensation (Merrill et al., 1999). Contrary to the function of the $S d$ allele, which effectively reduces the transmission of wild-type alleles to zero, the $b l$ allele results in a deficiency of $b l b l$ progeny when used as a female in crosses with $B l B l$ males. In addition, when $b l b l$ plants are used as males, an excess of blotchy-rooted progeny was detected. Thus, transmission ratio distortion was

thousands to millions of 180- and 350-base pair tandem repeats (Buckler et al., 1999). For many years, chromosome knobs have been associated with meiotic drive in maize. Meiotic drive in maize is due to interaction between meiotic drive genes and an abnormal version of a chromosome, known as Ab10 (Rhoades, 1952). Chromosome knobs may have evolved in response to meiotic drive, and they are ubiquitous in the maize genome. Buckler et al. (1999) demonstrated that maize evolution may have been significantly affected by structural changes in the genome, such as chromosome knobs, brought about by meiotic drive.

In many cases in plants, aberrant segregation ratios have been attributed to the effects of organellar genes. Cytoplasmic genes, including those in the mitochondria and chloroplast, can show strictly maternal, paternal, or both maternal and paternal transmission, thereby affecting segregation ratios. Little is known regarding the magnitude and mechanism of segregation distortion in most crop plants.

Several possible explanations for the observed segregation distortion may be offered. The $b l$ allele is responsible for blotchy patterning in the root of table beet plants, such that irregular sectors of betalain pigment are present in an unpigmented background (Watson and Goldman 1997). Betalain pigments likely have no demonstrable function in the roots of table beet plants and may simply be present due to human preference for (and selection of) root color during crop domestication. Leaves of $b l b l$ plants do not exhibit any obvious differences in betalain pigment patterning in leaves, and direct comparisons of betalain pigment concentrations in leaves and roots have not been made. If betalain pigments were associated with antioxidant properties and if $b l b l$ plants exhibited reduced amounts of pigment, the $B l$ allele might confer additional protection from photooxidation and thereby increase the fitness of $B l B l$ plants. However, this explanation is inadequate in explaining why an excess of blotchy-rooted plants were present in matings where blbl plants were used as males.

A second, and more likely, explanation is that the $b l$ allele may be linked to a gene associated with viability, thereby affecting the survivability and/or fitness of $b l b l$ plants. If linkage to viability genes is considered, the fitness of blbl plants may differ depend- 
ing on the direction of the cross from which they were produced. Blotchy-rooted plants derived from $b l b l \times B l B l$ matings were fewer in number than red-rooted plants from these matings, and more abundant than red-rooted plants in $B l B l \times b l b l$ matings. Goldman and Austin (2000) described linkage relationships among the $R, Y$, and $b l$ genes in table beet. They estimated linkage between the $R$ and $Y$ loci at $7.4 \pm 1.7 \mathrm{cM}$, and between $R$ and $b l$ at $16.7 \pm 10.8 \mathrm{cM}$, with a likely gene order of $R-Y$ - $b l$. If the $b l$ gene is linked to a locus associated with plant viability, additional investigation of this color gene complex may shed light on transmission ratio distortion in table beet.

The interaction of chromosome knobs with the abnormal version of chromosome 10 (Ab10) has been shown to cause meiotic drive in maize. Buckler et al. (1999) demonstrated a drive locus on Ab10 may have caused evolution of knobs in the maize genome, which in turn may have fostered meiotic drive. Knowledge of chromosome structure is limited in most major crop plants, with the exception of maize and wheat (Triticum aestivum L.). Little if anything is known concerning the presence of chromosome knobs and meiotic drive loci in Beta L. crops. However, if chromosome knobs are present in table beet, these may be associated with segregation distortion such as that measured in this study. Alternatively, if the blotchy locus were paramutable, conversion of $b l$ alleles to wild-type alleles could also account for the excess of red-rooted progeny in crosses where blbl plants such as that measured in this study can be adequately tested.

Findings from this investigation demonstrate transmission ratio distortion at the $b l$ locus in table beet. The $b l$ locus is linked to the $R$ and $Y$ loci, which condition betalain pigment distribution in the table beet plant. It is possible that the distortion measured in these populations is the result of linkage between the $b l$ locus and meiotic drive genes. If a gene conditioning meiotic drive were also segregating in these $\mathrm{F}_{2}$ populations, it is possible that the genotypes at this locus could explain the variability in segregation ratios among crosses observed herein. The transmission ratio distortion resulting in an excess of red-rooted progeny was greater (70\% as compared to $20 \%$ ) in crosses where blbl plants were used as females, however, an excess of red-rooted progeny was noted in both directions of the cross. If meiotic drive genes were present in table beet prior to the $b l$ mutation and are both linked to the $b l$ locus, and segregating in this cross, it is possible that ratio distortion could be observed when $b l b l$ plants are used as either males or females.

\section{Literature Cited}

Buckler, E.S., T.L. Phelps-Durr, C.S.K. Buckler, R.K. Dawe, J.F. Doebley, and T.P. Holtsford. 1999. Meiotic drive of chromosomal knobs reshaped the maize genome. Genetics 153:415-426.

Ganetzky, B, 1999. Yuichiro Hiraizumi and forty years of segregation distortion. Genetics 152:104.

Goldman, I.L. and D. Austin. 2000. Linkage among the $R, Y$, and $b l$ genes in table beet. Theor. Appl. Genet. 100:337-343.

Hollick, J.B., J.E. Dorweiler, and V.L. Chandler. 1997. Paramutation and related allelic interactions. Trends in Genet. 13:302-308.

Jones, R.N. 1991. B-Chromosome drive. Amer. Naturalist 137:430442.

Lyttle, T. 1991. Segregation distorters. Annu. Rev. Genet. 25:511-557. Lyttle, T. 1993. Cheaters sometimes prosper: Distortion of mendelian segregation by meiotic drive. Trends in Genet. 9:205-210.

Merrill, C, L. Bayraktaroglu, A. Kusano, and B. Ganetzky. 1999. Truncated RanGAP encoded by the segregation distorter locus of Drosophila. Science 283:1742-1745.

Patterson, A.H., J.W. DeVerna, B. Lanini, and S.D. Tanksley. 1990. Fine mapping of quantitative trait loci using selected overlapping recombinant chromosomes in an interspecific cross of tomato. Genetics 124:735742.

Rhoades, M.M. 1952. Preferential segregation in maize, p. 66-80. In: J.W. Gowen (ed.). Heterosis. Iowa State College Press, Ames.

Sandler, L. and E. Novitski. 1957. Meiotic drive as an evolutionary force. Amer. Naturalist 41:105-110.

Tsujimoto, H. and K. Tsunewaki. 1985. Gametocidal genes in wheat and its relatives. II. Suppressor of the chromosome $3 \mathrm{C}$ gametocidal gene of Aegilops triuncialis. Can. J. Genet. Cytol. 27:178-185.

Watson J.F. and I.L. Goldman. 1997. Inheritance of a recessive gene conditioning blotchy root color patterning in Beta vulgaris. J. Hered. 88:540-543.

Zamir, D. and Y. Tadmor. 1986. Unequal segregation of nuclear genes in plants. Bot. Gaz. 147:355-358. 